\title{
Active infrared sensing of impact damage in carbon fibre reinforced polymer
}

\author{
by Tong Kuan Chuah*, Liping Zhao*, Shaochun Ye*, Kwek Tze Tan**, \\ Pramoda Kumari Pallathadka**, Hui Ru Tan**
}

\author{
* National Metrology Centre, 1 Science Park Drive, Singapore 118221, chuah_tong_kuan@nmc.a- \\ star.edu.sg, zhao_liping@nmc.a-star.edu.sg, ye_shaochun@nmc.a-star.edu.sg \\ ** Institute of Materials Research and Engineering, 3 Research Link, Singapore 117602.
}

\begin{abstract}
With the growing demand of carbon fibre reinforced plastic (CFRP) in aerospace, marine and automobile industries, much attention is devoted to characterizing the material strength and characteristics of failure. This paper demonstrated the feasibility to estimate the internal damage non-destructively as a result of a quantified impact applied on 16-ply fabric CFRP. On thermography images at different heating time, differences were observed between intact area and area with internal damage. The estimation of 1D extent of damage using thermographic analysis was compared with images observed with cross sectional microscopic. The results suggest that qualitative analysis using thermography shows potential to be used as a tool for measuring impact damage.
\end{abstract}

\section{Introduction}

Carbon fibre reinforced plastic (CFRP) is widely used in the aerospace industry due to its high strength, and light weight [1, 2]. Impact can happen during takeoffs and landings, collisions with foreign objects (such as birds, stone etc.) or dropping of tools during maintenance. These events can affect the structural integrity of CFRP components. Therefore inspecting the internal damage of CFRP is important. However, a drawback of CFRP is that its impact failure is often internal and invisible [3]. As the use of CFRP is increased, there will be increasing need for its maintenance and inspection. Non-destructive testing (NDT) of CFRP such as using ultrasonic scanning based on attenuation has been developed [4] and used in practice with some inherent limitations such as need of couplant and limited penetration of ultrasound through superficial air gap. Meanwhile, developments of various other methods are underway to address the application issues such as that of in-situ operation, speed, sensitivity and contactless inspection. NDT methods being studied in the literature include Xray (projection and computed tomography), terahertz, and shearography. Infrared thermography is regarded as an attractive alternative for detecting and inspecting defects such as porosity and impact damage because of its non-contact nature, portability, non-ionizing light source and area-based inspection. Non-contact and non-ionizing nature provides assurance of not changing the nature of specimen, whereas portability facilitates ease of use insitu. Area based inspection is an advantage over point-based scanning method because it removes the need to scan point by point, which is time consuming. In a thermal transfer process, air gap and resin would give different thermal signals because they have different thermal properties. A well-developed inspection thermography system could potentially resolve impact damage from intact material effectively and efficiently, while also provide an estimate of size of the damage.

To study the feasibility of inspecting impact damage using thermography, we conducted experiments on CFRP materials with and without carbon nanotube (CNT) fillers. Our preliminary study dealt with qualitative evaluation of the infrared imaging system to visualize internal anomaly of CFRP such as thin film and hammering-induced damage. In the preliminary study, there are 4 pieces of samples, 2 of which are not filled (neat) with CNT and 2 of which are filled. One piece of the neat samples were ingrained with thin film and hammered; whereas one piece of CNT sample were hammered. These 4 samples were then observed under transient dynamic continuous heating process. Fig. 1 shows some results from the infrared images and processed images. Thin film inclusion was observed clearly in long wave infrared image and mid-wave infrared images upon heating using a halogen lamp. However, there is no clear indication of impact-induced contrast in the particular sample. The hammered CNT-filled samples showed some clear contrast as a result of hammering. Another observation is that mid-infrared camera does not give significantly better contrast nor details of the damage. These preliminary results suggest that it is feasible to use uncooled long wavelength infrared camera to evaluate impact-induced internal damage, and a quantitative and systematic study is worthy to further explore the capability in impact damage detection. 


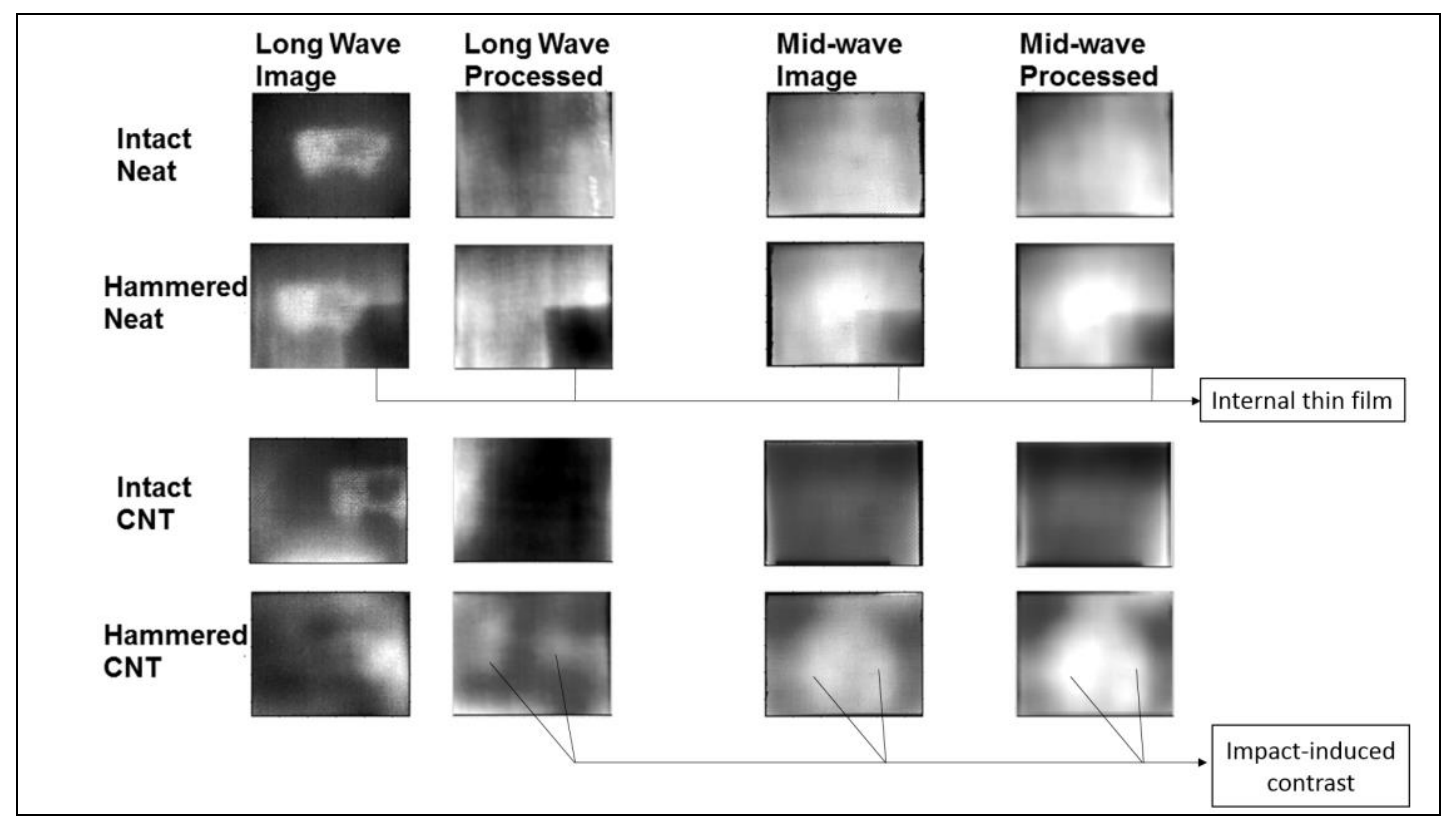

Fig. 1. Preliminary observations in infrared images for impact-induced internal damage, and thin film inclusion.

\section{Methods}

\section{Specimen and Impact Test}

Three pieces of CFRP fabric laminate samples (A1, B1, A2) were fabricated and two of them applied with controlled impact energy of 10 Joules under low velocity impact testing. The impact testing experiments were carried out using Instron Dynatub 9250HV Drop-Weight Impact Test Machine with a $5.57 \mathrm{~kg}$ steel tip impactor as shown in Fig. 2. Sample A1 is not applied with any impact and acted as a control sample. The size of each piece of sample is $65 \mathrm{~mm} \times 65 \mathrm{~mm}$ with a thickness in the range of 2.16 to $2.52 \mathrm{~mm}$. All samples have 16 plies of carbon fibre woven fabric and were made under the same conditions. The impactor has a line contact of $15.9 \mathrm{~mm}$ long formed between rounded impactor tip and the sample. During the impact testing, the sample is fixed by rubber clamp and supported firmly on a surface with a hole beneath the sample at the position of the impactor.

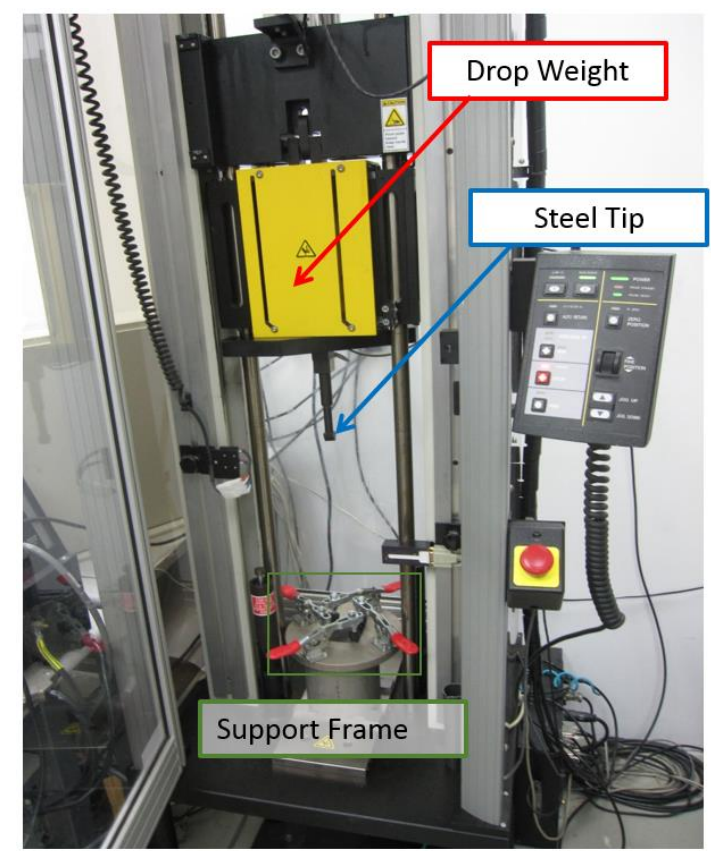

Fig. 2. Impact testing machine and its parts used for applying certain impact. 


\section{Thermography and Image Analysis}

The three pieces of samples were put side by side and on the same plane to be imaged by an uncooled long-wave infrared camera that covers the wavelengths from 7 to $12 \mu \mathrm{m}$. Fig. 3 shows the visual images of the 2 pieces of samples after applying impact. The samples were labelled A2 and B1 respectively (whereas A1 is the control sample, visual image not shown). These two impacted samples show some surface damage. Fig. 4 shows schematically the experimental setup for acquisition of the infrared images.

Once the acquisition was started, the halogen lamp was turned on to heat the 3 pieces of samples simultaneously and images were continuously acquired for the entire process of heating. The heating time for each experiment was 6 seconds and the experiments were repeated with the back faces (the face not in direct contact with impactor) of the samples facing the camera. The contrasts on each sample and between samples were observed. When the images show high contrast at certain time points, they were selected to be used for estimating the internal damage.

In this paper, we examine and validate the damage of a cross section from the top to the bottom in the middle of sample A2. Profiles of processed image (the processing involved spatial filtering, temporal filtering and taking the difference from initial image) were plotted and the extent of damage was estimated. The estimation was done by taking the mid-point of a local peak-valley separation (from the processed image) as the threshold to distinguish damage from intact portion. Taking the mid-point of peak-valley is an unbiased method to separate the low intensity caused by the damaged portion and an intensity peak. The intensity peak is formed by the high thermal transmission through the intact region, along with decreased intensity caused by (in addition to the damaged portion) the cold portion near the bottom of the sample due to thermal transmission to the clipping fixtures. Using the mid-point of this peak-valley separation although is unbiased, it is not particular to any physical damage, and it is not an empirically verified strategy and its sensitivity to internal damage is unknown, hence shall be examined in this and further related studies.
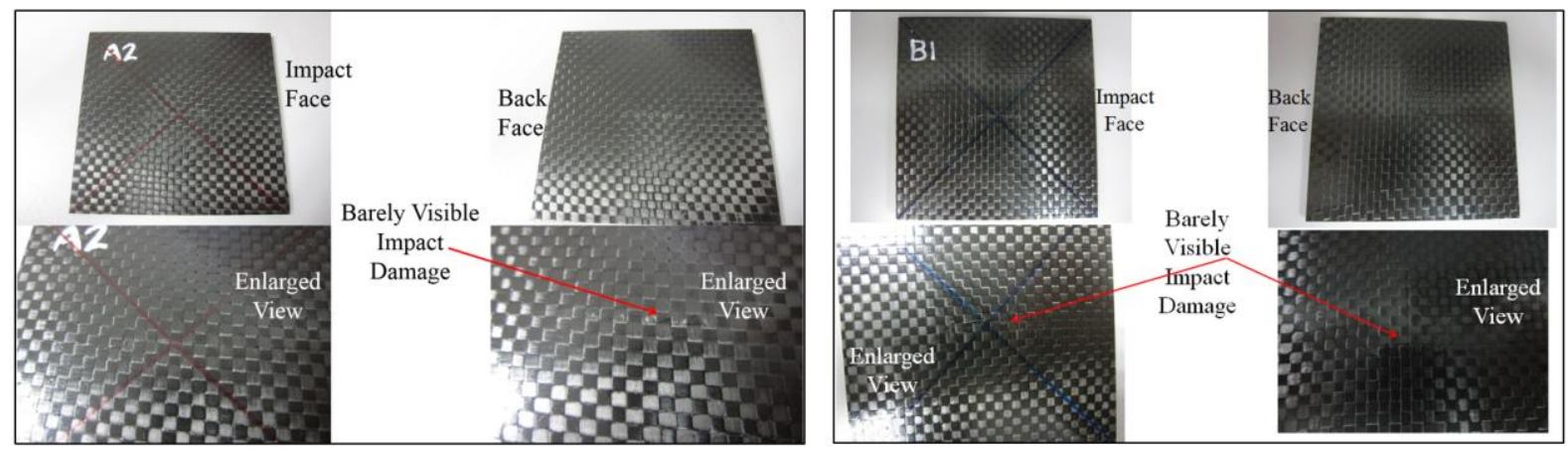

Fig. 3. Visual images of the samples applied with measured energy of $10 \mathrm{~J}$ each.

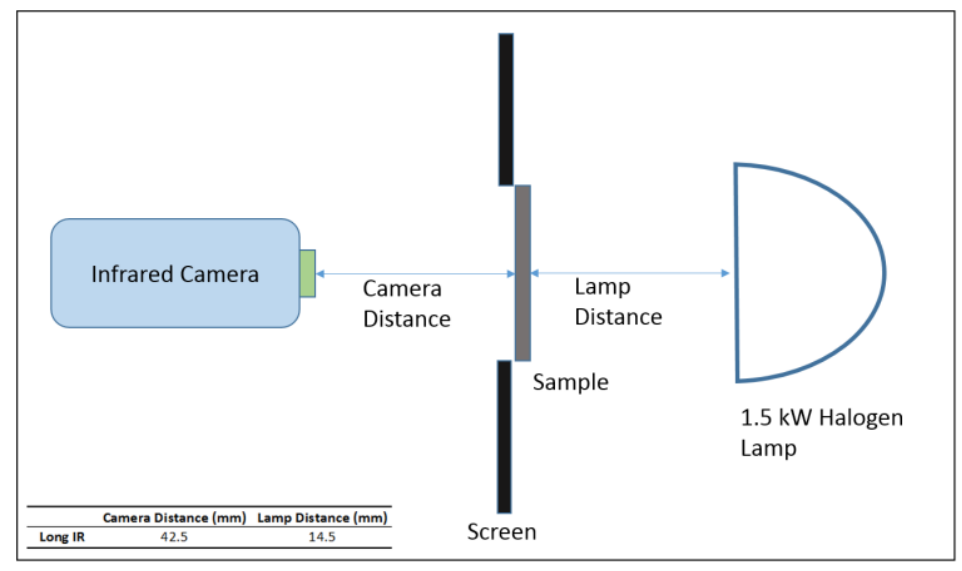

Fig. 4. Schematic diagram of experimental setup (top view). 


\section{Validation with Cross Sectional Microscopic Images}

After the infrared images were acquired, sample A2 was cut using a diamond wafering blade in its middle, as shown in Fig. 5. Microscopic images were observed and captured for analyzing the extent of damage portion along this line.

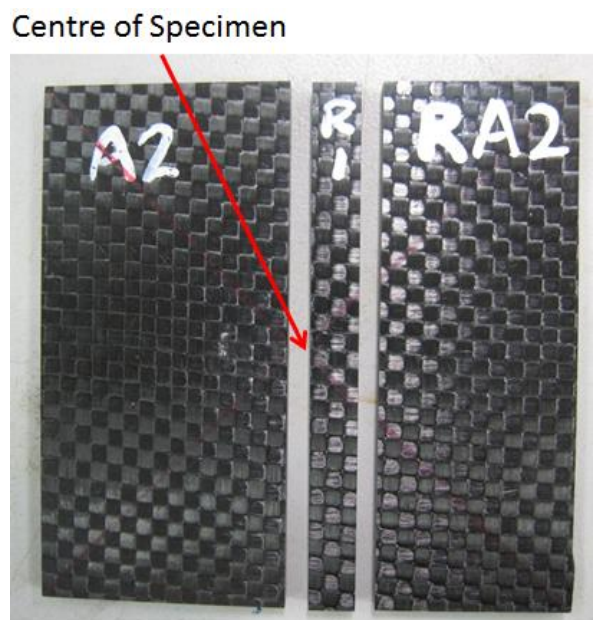

Fig. 5. Physical cut of sample observed with cross sectional microscopy.

\section{Results}

\section{Temperature Evolution}

Fig. 6 and Fig. 7 show the infrared (temperature) images at different time points. Good contrast can be observed between the expected damaged area and the intact area. These images are shown with varying greyscale scaled to the range of the individual 2D image. The images in Fig. 6 were taken while the camera is facing the face which had been contacted directly with the impactor, whereas those in Fig. 7 were images taken with the opposite side of the samples facing the camera.

For both impact face and the back face the change of images over time is in the following sequence: no contrast, good contrast and then low contrast, as can be expected from a dynamic heating cycle which a sample is changed from thermal equilibrium at room temperature and going towards (but not reaching) thermal equilibrium at higher temperature powered by the lamp.

Repeated comparison of the infrared images at different frames reveals that the best contrast appeared at $2.4 \mathrm{~s}$ at the impact face, but for the back face the best contrast appeared at $4.2 \mathrm{~s}$. Note that the contrast observed here is the difference between expected area of damage and expected intact area, proportionate to the range of grey level for each image at each particular frame. The contrast observed here is not the absolute grey level between the areas. The choice of using proportionate contrast instead of absolute contrast is an empirical one, and is also subjective to judgement. The subjectivity is an area to be improved upon. It is also observed that the thermal contrast patterns appeared at the two different faces are similar for the impacted samples, but the dark areas representing the damage appeared larger in the back face; whereas for the intact sample, no contrast is seen within the sample itself (other than cold region at the bottom due to thermal transfer towards clipping fixtures, and some possible leakage of light between samples). All these contrasts indicate that there is internal damage around the externally visible damage area.

The results suggest that thermography is able to identify internal damages in CFRP which are not visible from the outside. It is also apparent that the off-centre regions have more larger area of contrast compared to the centralized region of impact. On one hand, this could be due to uneven force distribution upon impact; and on the other hand, if we notice the pattern of spread of internal damage (small arrows in Fig. 8) there were damage propagations happened around the 2 corners of the impactor. There is some difference between the best contrast image on the front face and that on the back face. This difference may have some linkage with the depth of the damage, and suggest that the damage may not be symmetrical across the thickness of the sample. 

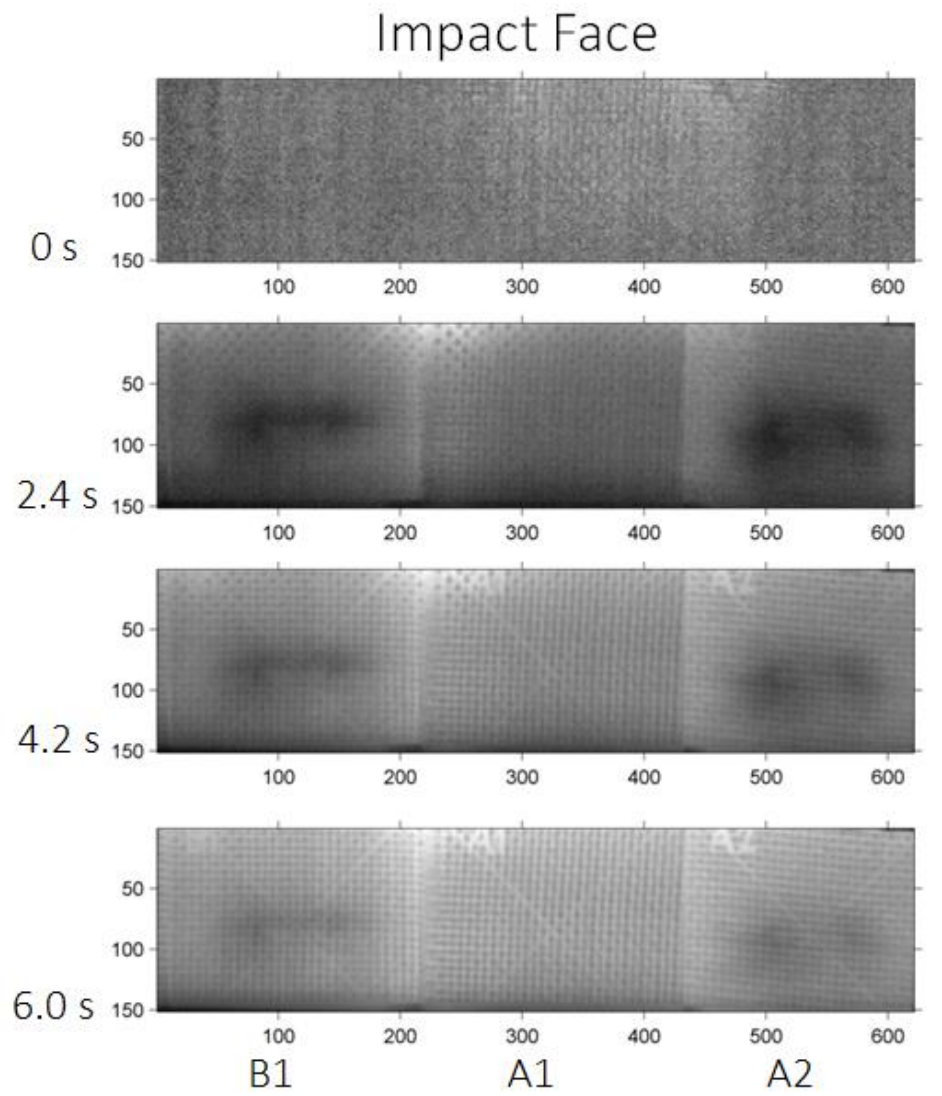

Fig. 6. Thermal images at different time during heating (camera facing impact face).
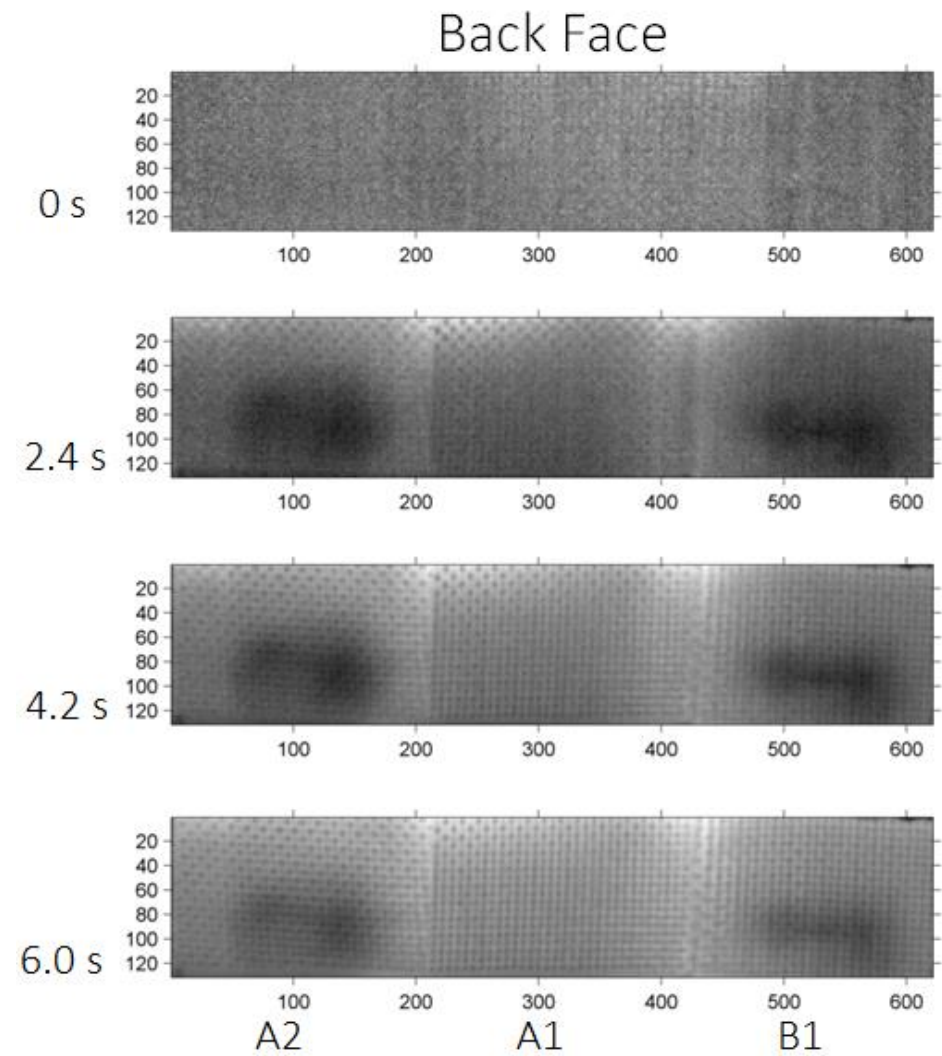

Fig. 7. Thermal images at different time during heating (camera facing opposite side of impact face). 


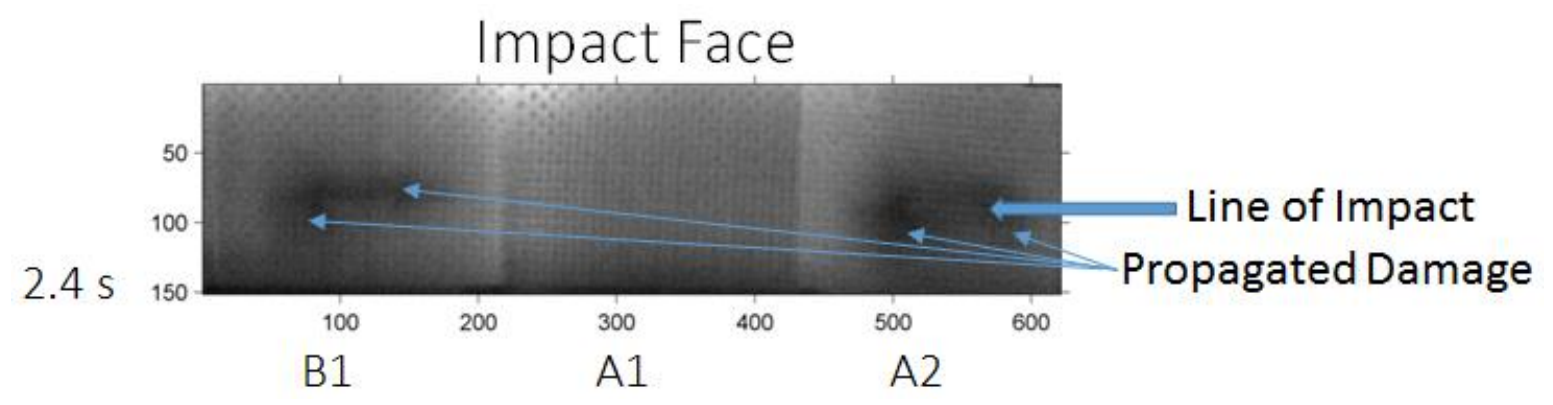

Fig. 8. Damage propagation adjacent to the line of damage

\section{Thermography Analysis of Extent of Damage}

Analysis was done for the front face thermograph using the difference from initial temperature (which is denoted as impact indicator) to estimate extent of damage for sample A2 comparing with the control sample and the results are shown in Fig. 9. The indicator is used instead of the raw temperature image because it has been found to provide images of less noise and information from surface reflection, all of which are undesired components for infrared image analysis. The estimated extent of damage is 34 pixels which correspond to 11 $\mathrm{mm}$. This number will be compared with the microscopic observation in the next subsection.

From Fig. 9, the threshold set using the local peak and local valley of the sample with $10 \mathrm{~J}$ impact is 2.078. If this value is plotted on the vertical profile of the control sample (bottom right of Fig. 9), the value only cut off the regions of the sample which are at the extreme bottom. The bottom parts of the samples were clipped by a set of polymer fixtures and thus appear colder than the other part of the sample; whereas the top part of the sample is in contact with air thus appear hotter. Given that the threshold only cut off the extreme of the control sample and does separate the expected damaged portion from the intact portion, it is deemed to be a reasonable choice of threshold. However, the thresholding method is dependent on the presence of intact area between the damaged area and the cold area which is at the bottom (resulted from the clipping fixtures). The method also may not cover all the damage present in the sample, therefore it can only be applied in practice if verified quantitatively with more samples.

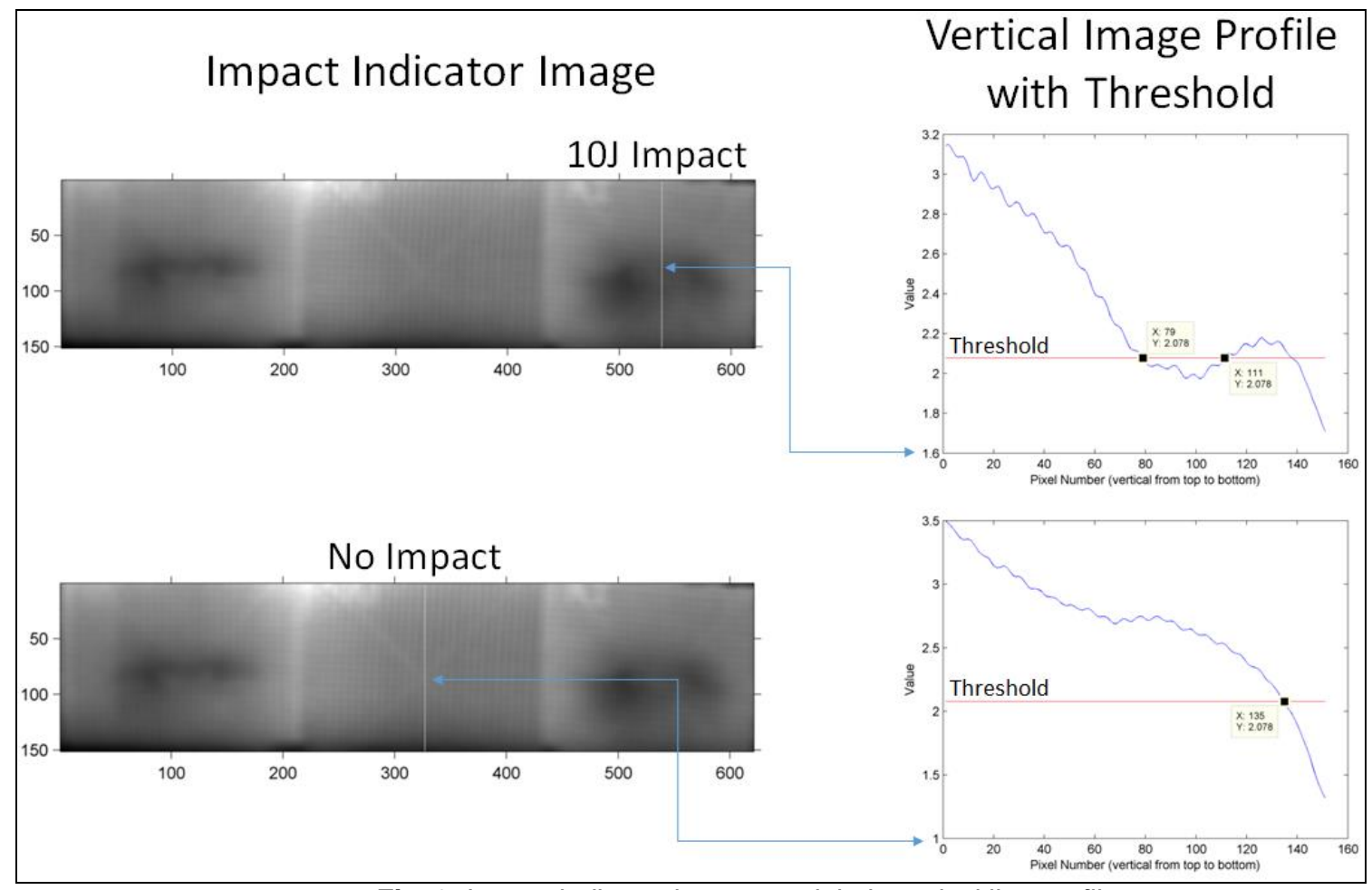

Fig. 9. Impact indicator images and their vertical line profile. 


\section{Cross Sectional Microscopy}

Microscopic images were captured at the centre of sample A2 down to $15 \mathrm{~mm}$ below its centre. Fig. 10a show the appearance of the sample at its centre, and Fig. 10b to $10 \mathrm{~d}$ show the damages observed at different locations below the centre of the sample.

The observation using microscopy has ascertained the presence of internal damage $10 \mathrm{~mm}$ below the centre of the specimen A2. The actual extent of damage is thus larger or equal to $10 \mathrm{~mm}$. In this sense, the finding of internal damage is consistent with that made by thermography analysis. If we consider the damage above the centre of the sample, it was observed to be approximately symmetrical with the damages below the centre, thus the actual extent of damage could be 20 to $30 \mathrm{~mm}$ long. Compared to $11 \mathrm{~mm}$ of estimation by thermography analysis, thermography therefore provided a lower reading for the extent of damage. Meaning, the analysis we used has detected the damage shown in Fig. 10b, but could not resolve the damage shown in Fig. 10c. The thermal analysis is therefore shown to have detected the damage of $400 \mu \mathrm{m}$ but unable to detect the damage of $200 \mu \mathrm{m}$. These results suggest that the sensitivity of the thermography system can still be further enhanced so that more damage can be detected. Approaches to enhance the sensitivity can be done through improvement of thermal sensing setup, improvement of experimental procedures and improvement of image processing methods such as the preprocessing and thresholding method. All these will be the focuses of the development of thermography as a method useful in actual application.

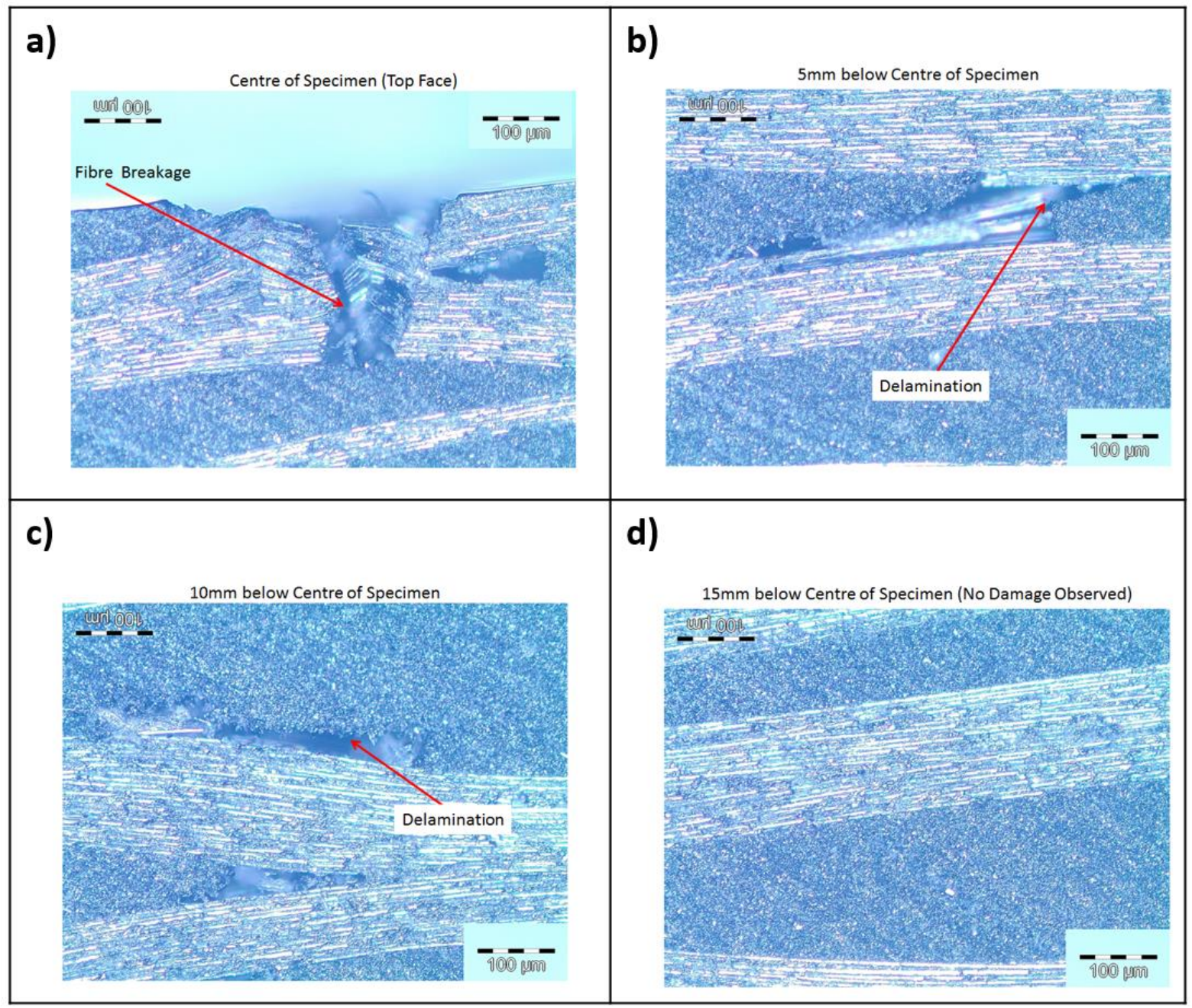

Fig. 10. Microscopic images at different locations, at and below the centre. 


\section{Conclusion}

This paper has demonstrated the feasibility of thermography to estimate internal impact damage of 16ply fabric laminate CFRP first by observation of the changes of temperature images, and then by threshold setting of the vertical profile on impact indicator image. It is observed that fibre breakage happened at the centre of the impact; and away from the centre, interfacial delamination occurred. Future work includes quantification of the entire area of damage by thermographic analysis and comparison of the estimated damaged area with that measured and/or observed using destructive testing. The sensitivity and spatial resolution of thermography for detecting internal damage shall be further studied.

\section{REFERENCES}

[1] Tang Y., Ye L., Zhang Z., Friedrich K., "Interlaminar fracture toughness and CAI strength of fibrereinforced composites with nanoparticles - A review”. Composites Science and Technology, vol. 86 pp. 26-37, 2013.

[2] Ye L., Lu Y., Su Z., Meng G., "Functionalized composite structures for new generation airframes: a review". Composites Science and Technology, vol. 65 pp. 1436-1446, 2005.

[3] Abrate S., "Impact on Laminated Composite Materials". Applied Mechanics Reviews, vol. 44, pp. 155190, 1991.

[4] Im K.H., Hwang Y.H., Song C.H., Kim T.H., Park J.W., Hsu D.K., Yang I.Y., "On fiber direction and porosity content using ultrasonic pitch-catch technique in CFRP composite solid laminates". $18^{\text {th }}$ International Conference on Composite Materials, 2011. 